\title{
Estimation and Minimization of the Cramer-Rao Lower Bound for Radio Direction-Finding on the Azimuth and Elevation of Planar Antenna Arrays
}

\author{
Y. Nechaev, and I. Peshkov
}

\begin{abstract}
In this paper an approach of obtaining optimal planar antenna arrays consisting of omnidirectional sensors is proposed. The novelty of the proposed approach is to apply an exact expression of the Cramer-Rao lower bound for an arbitrary planar antenna array consisting of a number of omnidirectional elements which has been presented in the further chapters of the paper. The obtained formula describes the influence of antenna elements locations on the direction-of-arrival estimation accuracy. It has been shown that the direction-of-arrival accuracy via planar antenna arrays is determined as the sum of squares of differences between all omnidirectional elements coordinates along $x$ - and $y$-axis. Thus knowing an expected area or sector of signal source it is very easy to calculate optimal arrangement of antenna elements in order to reduce directionfinding errors, because obtained by that way positions gives the best match according to the maximum likelihood criterion. It is worth nothing that such antenna arrays are useful in the way that they allow estimating the coordinates of radio emission sources in the three-dimensional coordinate space, i.e. in azimuth and elevation. In order to confirm the proposed methodology optimal antenna arrays constructed after minimization of the new formulas are researched. It is found out that the new shapes of antenna arrays based on the analytical expressions have better direction-of-arrival accuracy in comparison with the circular ones.
\end{abstract}

Index Terms-Antenna arrays, Phased arrays, Omnidirectional antennas, Direction-of-arrival estimation, Estimation error.

\section{INTRODUCTION}

$\mathrm{D}$ irection-of-arrival estimation (DOA) followed by spatial separation of users signal sources by antenna arrays (AA)

Manuscript received December 13, 2018; revised August 25, 2019. Date of publication November 14, 2019. Date of current version November 14, 2019 The associate editor prof. Giovanni Giambene has been coordinating the review of this manuscript and approved it for publication.

The work is supported by the Russian Science Found. The project number is $18-71-00080$

Yuri Nechaev is with Department of Information Systems, Voronezh State University, Universitetskaya sq., 1, 394000, Voronezh, Russia (e-mail: nechaev_ub@mail.ru).

Ilia Peshkov is with Department of Physics, Radio engineering and Electronics, Bunin Yelets State University, Ordzhonikidze st., 79, 399770 Yelets, Russia (e-mail: ilvpeshkov@gmail.com).

Digital Object Identifier (DOI): 10.24138/jcomss.v15i4.672 takes a great interest in such tasks as radars, sonars and wireless communications in order to increase the capacity of the network with a dynamically changing radiation pattern by means of the beamforming algorithms. Today a variety of direction-finding methods with superresolution capable of resolving signals in the resolution interval according to the Rayleigh criterion have been distributed, such as projections onto subspaces, maximum likelihood, etc., and also digital beamforming algorithms that are the optimal spatial filters of only linear antenna arrays with ideal non-directional emitters. The antenna arrays configurations which were researched and used for DOA-estimation on the azimuth plane mainly concerned only uniform linear geometries [1-6]. Moreover, it is well known that antenna array geometry imposes some limitations on the direction-finding measurements, like only azimuth estimation capability [7]. The circular and concentric antenna array is used to overcome the problem of this kind in the tasks requiring both azimuth and elevation directionfinding [8-10]. Thus the most popular configurations of the antenna arrays are uniform linear, rectangular, as well as uniform circular ones which had been investigated mainly in the azimuth plane assuming that the antennas are isotropic [110]. Therefore, the problem of comparative analysis, research and development of methods for spatial filtering of wideband signals in the azimuth and elevation planes using planar systems (rectangular, circular, concentric, etc.), as well as spherical and other variants of conformal antenna arrays represents a serious research interest. Thus the paper is devoted to obtaining planar antenna array with higher accuracy by using the direction-of-arrival methods like MUSIC or Capon. That can be develop[ed further for the digital beamforming. Moreover, the accuracy is determined quantitatively, either as the root mean square error rate of the bearing estimates in azimuth or elevation from their true values, or as the variance.

The Cramer-Rao lower bound is a natural and common criterion for choosing geometry of antenna arrays, which does not depend on the choice of one or another algorithm for implementing the evaluation of the spatial angular coordinates of signals on the azimuth or elevation planes. In papers [1112] the problem of obtaining the Cramer-Rao lower bound 
(CRLB) for the azimuth and elevation DOA-estimation using antenna arrays with omnidirectional elements is considered. They make the use of the polar coordinates of the array sensors and do not give an exact expression taking into account the position of the source together with the locations in space of each antenna element. The approach does not allow accurately assessing the antenna elements positions impact on DOA-estimation performances and build an array with the best performance within confined sectors.

The following is an improved and expanded matrix-vector expression of the Cramer-Rao lower bound taking into account the aforementioned shortcomings. In particular, the new generic expression of CRLB takes into account only the coordinates in the Cartesian system, which is natural to realize and also allows evaluating the mutual influence of the positions of elements on the accuracy of direction-finding of any array geometry. Additionally, the obtained equation will be very helpful to analyze the important factors which determine the DOA-estimation accuracy by using the antenna arrays of these kinds. Impact on these factors will help us to generate such configuration that will have better characteristics for accuracy and resolution of DOA methods.

Several different performance and design criteria have been introduced to be used in obtaining optimal arrays [13-17], we can say the array with the highest bound is optimum in the sense that array is constructed using prespecified performance levels, in our case Cramer-Rao Bounds on error variance and minimum and maximum coordinates in the $X Y$ plane. The calculations can be executed manually by means of the presented in the paper simple relationships. Finally, we provide simple formulas for comparing the performance of different arrays to each other including optimal ones. The proposed array design does not take into account array ambiguity as in [18].

The paper is organized as follows. The Chapter II provides an overview of related works. In the chapter III the well known representations of narrowband signals and antenna arrays are described. In the chapter IV CRLB for DOAestimation on azimuth-elevation is discussed [10]. Chapter V gives the previously obtained results in [8] about symmetric antenna arrays and their CRLB values. Part VI discusses nonsymmetric antenna arrays that have not previously been published, but it is a continuation of Part $\mathrm{V}$ and also a special case. Parts VII-VIII present the derivations of the general expression of CRLB for arbitrary two- and three-element planar arrays describing how the DOA accuracy is related with the $x$ and $y$ coordinates of the elements. Part IX gives the general expression of CRLB for an arbitrary planar array out of any number of elements. Examples of obtaining new arrays reducing DOA errors are given.

\section{RELATED WORK}

There are several criteria for statistical evaluation of parameter estimates: the Ziv-Zakai bound, the CRLB etc. The Ziv-Zakai bound is a detection probability, while CRLB belongs to so-called inequality bounds [19, 20]. Historically, the latter is most widely used for DOA-estimation.

Cramer-Rao bound on direction of arrival estimation have been thoroughly analyzed in the literature. As it was mentioned above there are several criteria which have been introduced in obtaining optimal AA. Among them it is possible to underline the paper in which the dispersion of a antennas distribution [21] is used for the optimization of the 2D geometry of sensor arrays for 2D direction-of-arrival (DOA) estimation. In other words the variance of the element positions is used for that. Another work is devoted to optimal antenna array geometry obtaining ant that can be fulfilled if using the complex number when denoting the antennas coordinates [22] in space. In the paper [22] the placement of the $m$ th antenna element is stated by a complex value. A lot of references are devoted to the antenna optimization by using the approximate expression of the Cramer-Rao bound like in [23; 24]. Based on the simplified CRLB equation the metric function is defined and minimized. A few papers consider only certain array shapes [13] like 3D particular geometry antennas made from uniform linear array (ULA). As it can be seen from the above, these approaches complicate the implementation of the search for the solution of cost functions, because it is necessary to use genetic algorithms. For this reason in the proposed method the AA will be considered an optimal with minimum error dispersion of the CRLB direction finding, under the condition that antenna element (AE) coordinates are restricted by the given bounds. It follows that another purpose of the work is to obtain a convenient target function in the form of the CRLB expression, dependent upon the AE coordinates along the $\mathrm{x}, \mathrm{y}$ and $\mathrm{z}$ axes.

The studies described in the references [11-12] consider the problem of obtaining the CRLB expression to estimate the angular coordinates of signal sources using AAs with omnidirectional sources. The distinctive feature of these studies is the use of AEs polar coordinates, which is unacceptable for the purposes at hand. The originality of the proposed method consists in minimizing the new generalized CRB expression, taking into account only the coordinates of the AEs in the Cartesian system, as well as allowing estimating mutual influence of AEs positions on the accuracy of radio direction finding within the arbitrary antenna configuration. The advantage of the proposed method reduces to the clarity and simplicity of the implementation, because optimization can be performed either manually or with the help of a well-known the steepest descents algorithm. Among other things, it is possible to introduce location restrictions on all elements, or on certain elements, for example, with the purpose of mounting the AA on any vehicle (a car, plane or train).

\section{PROBLEM ForMULATION}

Fig. 1 shows an antenna array of $N$ elements arbitrarily distributed in the $X Y$ plane. Consider a narrow-band signal $s(t)$ at a carrier frequency $\omega_{0}$ with spatial coordinates $\theta$ (azimuth) 
and $\varphi$ (elevation) with respect to the $x$ and $z$ axes [4]:

$$
\tilde{s}(t)=u(t) \cos \left(\omega_{0}+v(t)\right)
$$

where $u(t)$ and $v(t)$ are slowly changing functions of time. As the signal is narrowband, then the delay $\tau_{\mathrm{i}}$ causes the phase shift $\xi_{i}=-\tau_{i} \omega_{0}$, i.e.

$$
\begin{gathered}
s(t-\tau)=s(t) e^{j \xi_{i}}=s(t) e^{-j \tau_{i} \omega_{0}} \\
\xi_{i}=\frac{\omega_{0}}{c}\left[x_{i} \cos \theta \sin \varphi+y_{i} \sin \theta \sin \varphi+z_{i} \cos \varphi\right]
\end{gathered}
$$

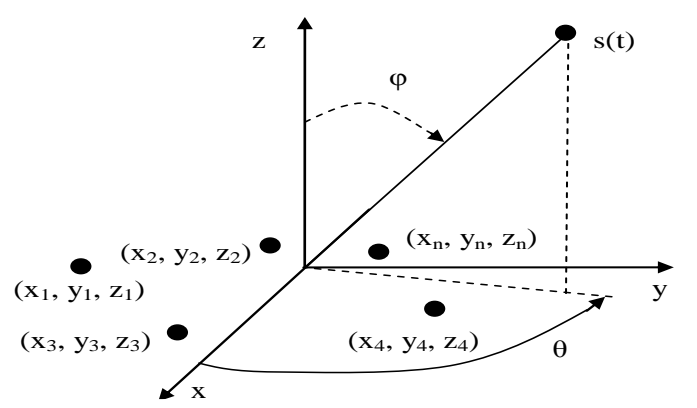

Fig. 1. Arbitrary planar antenna array.

where $\lambda$ is the wavelength. And now, if the signals on the antenna elements are described as $\mathbf{x}_{1}, \mathbf{x}_{2}, \ldots, \mathbf{x}_{\mathrm{N}}$, then in the vector notation they will look like:

$$
\left.\mathbf{x}(t)=\mathbf{a}(\omega, \theta, \lambda) s(t)=l_{e^{j \mathbf{k} \mathbf{r}_{1}^{T}}}^{e^{j \mathbf{k} \mathbf{r}_{2}^{T}}} \cdots \cdots e^{j \mathbf{k r}_{N}^{T}}\right\rfloor_{s}(t)
$$

where $\mathbf{k}=\frac{2 \pi}{\lambda}\left(k_{x}, k_{y}, k_{z}\right)=(\cos \theta \sin \varphi, \sin \theta \sin \varphi, \cos \varphi)$ is the wave number, $\mathbf{r}_{n}^{T}=\left(x_{n}, \mathrm{y}_{n}, \mathrm{z}_{n}\right)^{T}$ is the radius-vector pointed to the $n$-th antenna element.

As already shown [25], the accuracy of direction-of-arrival estimation is influenced by the inter-element spacing, as well as the shape of the antenna array. A natural and common criterion for choosing the antenna array geometry is the Cramer-Rao lower bound. The antenna elements in all the arrays are omnidirectional sensors. The geometry and the received voltage expressions of each antenna array are introduced in this Section, which are prepared for the CRLB calculation.

The geometry of square, hexagonal, octagonal and circular symmetric planar antenna arrays are shown in Fig. 2. The received voltage of square array is

$$
a_{m n}^{s q}(\phi, \theta)=\exp \left\{j k\left[(n-1) d_{x} k_{x}+(m-1) d_{y} k_{y}\right]\right\}
$$

where $1 \leq n \leq N_{x}, 1 \leq m \leq N_{y}, N x$, and $N y$ are the antenna element numbers along $X$ and $Y$ axis, $d x$ and $d y$ is the interelement distance along the $X$ and $Y$ axis.

The steering vector value of circular antenna array:

$$
a_{n}^{\text {circ }}(\phi, \theta)=\exp \left\{j\left[-k r \cos \left(\theta-\frac{2 \pi n}{N}\right) \sin (\phi)\right]\right\}
$$

where $r$ is the antenna array radius, $n=1 \ldots N, k=2 \pi / \lambda$.

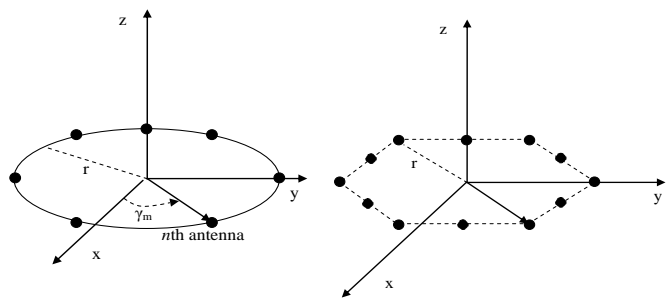

a)

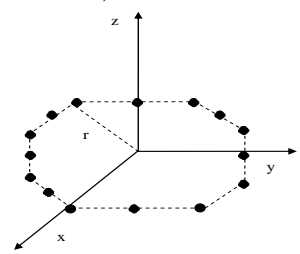

c)

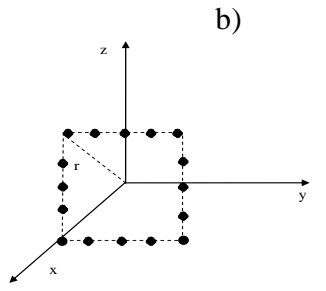

d)
Fig. 2. Geometries of a) circular, b) octagonal, c) hexagonal, d) square arrays

The views of hexagonal and octagonal arrays are illustrated in Fig. 2 b-c and their voltage values, which can be obtained from (1), are written as:

$a_{m n}^{h e x}(\phi, \theta)=\exp \left\{j k\left[\left(n-\frac{N_{x}-|m|-1}{2}\right) d_{x} k_{x}+\frac{\sqrt{3}}{2} m d_{y} k_{y}\right]\right\}$

$a_{m n}^{o c t}(\phi, \theta)=\exp \left\{j k\left[\left(n-\frac{N_{x}-|m|-1}{2}\right) d_{x} k_{x}+\frac{\sqrt{2}}{2} m d_{y} k_{y}\right]\right\}$

where

$$
m=-\frac{N_{x}-1}{2},-\frac{N_{x}-1}{2}+1, \ldots, \frac{N_{x}-1}{2},
$$

$n=0,1, \ldots, N_{x}-|m|-1$. All the equations of received voltages are obtained after solving and simplifying $\mathbf{k r}_{n}^{T}$ in (4) taking into account position of each antenna element.

\section{CRAMER-RAO LOWER BOUND FOR DOA}

The Cramer-Rao lower bound, related to the concept of Fisher Information, represents the theoretical limit of the average precision with which DOA can be estimated for a particular antenna array. Suppose $\hat{\boldsymbol{\eta}}$ is unbiased estimation of the parameter vector $\boldsymbol{\eta}_{0}$, i.e. $E\{\hat{\boldsymbol{\eta}}\}=\boldsymbol{\eta}_{0}$ based on samples $\mathbf{X}_{N}$, then the derivation of the Cramer-Rao lower bound to an arbitrary number of signals and their parameters (first of all, azimuth and angle direction finding) we use [15]. We define the matrices of the derivatives $\mathbf{D}_{\theta}$ and $\mathbf{D}_{\varphi}$ as follow:

$$
\mathbf{D}_{\theta, \varphi}=\left[\left.\frac{\partial \mathbf{a}\left(\theta_{1}, \varphi_{1}\right)}{\partial \eta}\right|_{\eta=\theta_{1}, \varphi_{1}}, \ldots,\left.\frac{\partial \mathbf{a}\left(\theta_{d}, \varphi_{d}\right)}{\partial \eta}\right|_{\eta=\theta_{d}, \varphi_{d}}\right]
$$

Thus, the covariance matrix of error estimates for $2 \mathrm{D}$ radio direction-finding can be written [16]: 


$$
\mathbf{B}_{\text {STO }}=\frac{\sigma^{2}}{2 N} \mathfrak{R}\left[\operatorname{Tr}\left\{\left[\begin{array}{ll}
\Lambda_{1} & \Lambda_{2} \\
\Lambda_{3} & \Lambda_{4}
\end{array}\right] \circ\left[\begin{array}{ll}
\Xi & \Xi \\
\Xi & \Xi
\end{array}\right]^{T}\right\}\right]^{-1},
$$

where $\quad \boldsymbol{\Lambda}_{1}=\mathbf{D}_{\theta}^{H} \mathbf{P}_{A}^{\perp} \mathbf{D}_{\theta}, \quad \boldsymbol{\Lambda}_{2}=\mathbf{D}_{\theta}^{H} \mathbf{P}_{A}^{\perp} \mathbf{D}_{\varphi}, \quad \boldsymbol{\Lambda}_{3}=\mathbf{D}_{\varphi}^{H} \mathbf{P}_{A}^{\perp} \mathbf{D}_{\theta}$, $\mathbf{\Lambda}_{4}=\mathbf{D}_{\varphi}^{H} \mathbf{P}_{A}^{\perp} \mathbf{D}_{\varphi}, \Xi=\mathbf{S} \mathbf{A}^{H} \mathbf{R}^{-1} \mathbf{A} \mathbf{S}$.

As we can see from the equation (10), the matrices $\boldsymbol{\Lambda}_{i}$ have to be specified by means of determining the partial derivatives of the steering vectors of an arbitrary antenna array (4) from $\theta$ and $\varphi$ :

$$
\frac{\partial \mathbf{a}\left(\theta_{m}, \varphi_{m}\right)}{\partial \eta}=\frac{\partial e^{j \mathbf{k}_{1} \mathbf{R}^{T}}}{\partial \eta}=\frac{\partial j \mathbf{k}_{1} \mathbf{R}^{T}}{\partial \eta} e^{j \mathbf{k}_{1} \mathbf{R}^{T}}
$$

First we define the derivative of exponential part on azimuth for $k$-th signal on $n$-th antenna element [8]:

$$
\begin{aligned}
& \frac{\partial e^{j \psi}}{\partial \theta_{k}}=\frac{\partial j \psi}{\partial \theta_{k}} e^{j \psi}= \\
& \frac{\partial j \frac{2 \pi}{\lambda}\left(x_{n} k_{x}+y_{n} k_{y}+z_{n} k_{z}\right)}{\partial \theta_{k}} e^{j\left(\frac{2 \pi}{\lambda}\left(x_{n} k_{x}+y_{n} k_{y}+z_{n} k_{z}\right)\right)}= \\
& j\left(\frac{2 \pi}{\lambda}\left(-x_{n} \sin \theta \sin \varphi+y_{n} \cos \theta \sin \varphi\right)\right) e^{j\left(\frac{2 \pi}{\lambda}\left(x_{n} k_{x}+y_{n} k_{y}+z_{n} k_{z}\right)\right)}
\end{aligned}
$$

Next, it is necessary to determine the expressions for the derivatives of exponential part on elevation spatial coordinate for the $k$-th signal on the $n$-th antenna element:

$$
\begin{aligned}
& \frac{\partial e^{j \psi}}{\partial \varphi_{k}}=\frac{\partial j \psi}{\partial \varphi_{k}} e^{j \psi}= \\
& \frac{\partial j \frac{2 \pi}{\lambda}\left(x_{n} k_{x}+y_{n} k_{y}+z_{n} k_{z}\right)}{\partial \varphi_{k}} e^{j\left(\frac{2 \pi}{\lambda}\left(x_{n} k_{x}+y_{n} k_{y}+z_{n} k_{z}\right)\right)}= \\
& j\left(\frac{2 \pi}{\lambda}\left(x_{n} \cos \theta \cos \varphi+y_{n} \sin \theta \cos \varphi-z_{n} \sin \theta\right)\right) e^{j\left(\frac{2 \pi}{\lambda}\left(x_{n} k_{x}+y_{n} k_{y}+z_{n} k_{z}\right)\right)}
\end{aligned}
$$

Then the Cramer-Rao lower bound for azimuth or/and elevation estimation can be written [21]:

$$
\left[\begin{array}{ccc}
\operatorname{var}\left(\theta_{1}, \varphi_{1}\right) & & 0 \\
& \ddots & \\
0 & & \operatorname{var}\left(\theta_{M}, \varphi_{M}\right)
\end{array}\right]=\frac{\sigma^{2}}{2 N} \Re\left[\left(\mathbf{D}_{\theta, \varphi}^{H} \mathbf{P}_{A}^{\perp} \mathbf{D}_{\theta, \varphi}\right) \circ \Xi\right]^{-1}
$$

As we can see the equation (13) is rather complicated and here we consider only one particular case. Then after simplifying (4), CRLB for the case of only one signal source can be expressed as follows:

$$
\operatorname{var}(\varphi, \theta)=\frac{\sigma^{2}}{2 K} \mathfrak{R}\left[\left(\frac{\partial \mathbf{a}^{H}}{\partial \eta}\left(\mathbf{I}-\mathbf{a}\left(\mathbf{a}^{H} \mathbf{a}\right)^{-1} \mathbf{a}^{H}\right) \frac{\partial \mathbf{a}}{\partial \eta}\right) \circ \Xi\right]^{-1}
$$

The equation (14) can be re-written more compactly as follows:

$$
\operatorname{var}(\varphi, \theta)=\frac{\sigma^{2}}{2 K P_{s}} \frac{1}{A R}\left(\frac{\lambda}{2 \pi}\right)^{2}
$$

Assuming that $\Xi \approx P_{S}$, i.e. the signal power in the one signal case, we can express the term inside square brackets in the equation (15) in the following matrix-vector form as [8]:

$$
\begin{gathered}
\frac{\partial \mathbf{a}^{H}}{\partial \eta}\left(\mathbf{I}-\mathbf{a}\left(\mathbf{a}^{H} \mathbf{a}\right)^{-1} \mathbf{a}^{H}\right) \frac{\partial \mathbf{a}}{\partial \eta}=\frac{\partial \mathbf{a}^{H}}{\partial \eta}\left(\mathbf{I}-N^{-1} \mathbf{a a}^{H}\right) \frac{\partial \mathbf{a}}{\partial \eta}= \\
\frac{\partial \mathbf{a}^{H}}{\partial \eta}\left[\begin{array}{cccc}
\frac{1-N}{N} & -\frac{1}{N} a_{1} a_{2}^{H} & \ldots & -\frac{1}{N} a_{1} a_{N}^{H} \\
-\frac{1}{N} a_{2} a_{1}^{H} & \frac{1-N}{N} & \ldots & -\frac{1}{N} a_{2} a_{N}^{H} \\
-\frac{1}{N} a_{N} a_{1}^{H} & -\frac{1}{N} a_{N} a_{2}^{H} & \ldots & \frac{1-N}{N}
\end{array}\right] \frac{\partial \mathbf{a}}{\partial \eta}
\end{gathered}
$$

Further we accept the following designations $a_{i} a_{i}^{H}=1$ and $a_{i}^{\prime}=\frac{\partial j \xi_{i}}{\partial \theta}$. After that the term $A R$ inside the equation (16) can be written as

$$
\begin{aligned}
A R= & 1 /\left(\frac{\lambda}{2 \pi}\right)^{2}\left[\begin{array}{lll}
\left(\mathbf{k}^{\prime} \mathbf{r}_{1}\right)^{H} \mathbf{k}^{\prime} & \ldots & \left(\mathbf{k}^{\prime} \mathbf{r}_{N}\right)^{H} \mathbf{k}^{\prime}
\end{array}\right] . \\
& {\left[\begin{array}{cccc}
\frac{N-1}{N} & -\frac{1}{N} & \ldots & -\frac{1}{N} \\
-\frac{1}{N} & \frac{N-1}{N} & \ldots & -\frac{1}{N} \\
-\frac{1}{N} & -\frac{1}{N} & \ldots & \frac{N-1}{N}
\end{array}\right]\left[\begin{array}{c}
\mathbf{r}_{1} \\
\ldots \\
\mathbf{r}_{N}
\end{array}\right] }
\end{aligned}
$$

As we can see the term $A R$ depends on the antenna array configuration only. So that the Cramer-Rao lower bounds is inversely proportional to the term $A R$. In order to make variance of DOA-estimation on the azimuth or/and elevation planes as lower as possible, $A R$ should be as large as possible. As we can see from the $A R$ equation (17), increasing the difference between the radius-vectors pointed to the antenna elements $\mathbf{r}_{n}^{T}=\left(x_{n}, \mathrm{y}_{n}, \mathrm{z}_{n}\right)^{T}$ will help to make the $A R$ term as maximum as possible.

\section{EXACT EQUATION FOR CRAMER-RAO LOWER BOUND FOR DOA VIA SYMMETRIC ARRAYS}

Here we can perform comparative statistical estimation of the planar antenna array based on the Cramer-Rao bound (10). The circular, octagonal, hexagonal and square antenna arrays 
will be researched further as follows in the Section. All the antenna arrays geometries consist of 24 elements, interelement spacing is $0.5 \lambda$ (half-wavelength). The number of snapshots of averaging the spatial correlation matrix is 100 , the number of trial iterations is 500 . We will be using the equation (1) to set up the signals matrix $\mathbf{S}$ and noise power $\sigma^{2}$ which define signal-to-noise ratio $(S N R)$ and then $S N R$ is $5 \mathrm{~dB}$. Here we assume that the signals are uncorrelated between themselves and the noise. The root mean square error rate of direction-of-arrival estimation on azimuth and elevation will be performed. Consider the case when there is a signal source, azimuth spatial coordinate will be changed from $0^{\circ}$ up to $180^{\circ}$, elevation angle $\theta=45^{\circ}$. In another case the elevation coordinate will vary in the range $1^{\circ}$ to $89^{\circ}$ at a fixed azimuth angle value of $1^{\circ}$.

However, first, it is necessary to determine the exact $A R$ expressions for the types of antenna arrays under consideration by using the equations $(16,17)$. At present there are a few ways and approaches to estimate antenna arrays for directionof-arrival estimation by means of the Cramer-Rao lower bound exact equation in known references, in particular [18,21]. However the CRLB described in the references is calculated only for one type of antenna array configuration (circular, as example) or for a limited number of elements (two, as example). Further, several symmetrical planar antenna arrays are considered, with the help of which it is possible to determine the coordinates along the azimuth and the elevation angles.

So, for a rectangular antenna array:

$$
\begin{gathered}
A R_{\theta}^{s q}=146\left(\frac{\lambda}{2} \sin \theta \sin \varphi\right)^{2}+146\left(\frac{\lambda}{2} \cos \theta \sin \varphi\right)^{2}, \\
A R_{\varphi}^{s q}=146\left(\frac{\lambda}{2} \cos \theta \cos \varphi\right)^{2}+146\left(\frac{\lambda}{2} \sin \theta \cos \varphi\right)^{2},
\end{gathered}
$$

here and further $A R_{\theta}$ and $A R_{\varphi}$ are the AR functions in the azimuth and elevation angle, respectively. Since only planar antenna arrays are considered in this paper, $z_{n}$ coordinates are assumed to be zero.

For the hexagonal antenna array:

$$
\begin{gathered}
A R_{\theta}^{\text {hex }}=162\left(\frac{\lambda}{2} \sin \theta \sin \varphi\right)^{2}+162\left(\frac{\lambda}{2} \cos \theta \sin \varphi\right)^{2}, \\
A R_{\varphi}^{\text {hex }}=162\left(\frac{\lambda}{2} \cos \theta \cos \varphi\right)^{2}+162\left(\frac{\lambda}{2} \sin \theta \cos \varphi\right)^{2} .
\end{gathered}
$$

For the octagonal antenna array:

$$
A R_{\theta}^{o c t}=168.4\left(\frac{\lambda}{2} \sin \theta \sin \varphi\right)^{2}+168.4\left(\frac{\lambda}{2} \cos \theta \sin \varphi\right)^{2},
$$

$$
A R_{\varphi}^{o c t}=168.4\left(\frac{\lambda}{2} \cos \theta \cos \varphi\right)^{2}+168.4\left(\frac{\lambda}{2} \sin \theta \cos \varphi\right)^{2} .
$$

For the circular antenna array:

$$
\begin{gathered}
A R_{\theta}^{\text {circ }}=176\left(\frac{\lambda}{2} \sin \theta \sin \varphi\right)^{2}+176\left(\frac{\lambda}{2} \cos \theta \sin \varphi\right)^{2}, \\
A R_{\varphi}^{\text {circ }}=176\left(\frac{\lambda}{2} \cos \theta \cos \varphi\right)^{2}+176\left(\frac{\lambda}{2} \sin \theta \cos \varphi\right)^{2} .
\end{gathered}
$$

So that, it is possible to make comparisons about the $A R$ parameter for the given arrays [8]:

$$
A R_{\varphi, \theta}^{s q}<A R_{\varphi, \theta}^{h e x}<A R_{\varphi, \theta}^{o c t}<A R_{\varphi, \theta}^{c i r c}
$$

and consequently

$$
C R L B_{\varphi, \theta}^{s q}>C R L B_{\varphi, \theta}^{\text {hex }}>C R L B_{\varphi, \theta}^{o c t}>C R L B_{\varphi, \theta}^{\text {circ }}
$$
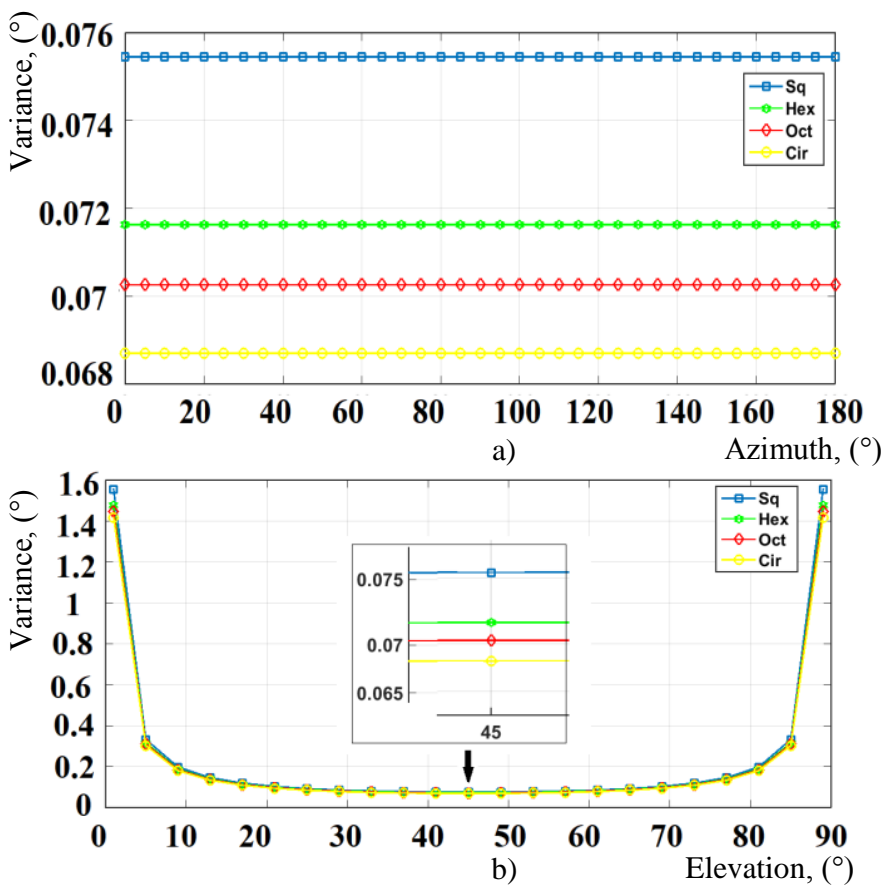

Fig. 3. Cramer-Rao bound a) $\theta=0^{\circ}-180^{\circ}, \varphi=45^{\circ}$, b) $\theta=1^{\circ}, \varphi=1^{\circ}-89^{\circ}$ for one signal case.

From Figure 3 it is seen that the best antenna array is circular for DOA-estimation. The antenna geometry with the worst accuracy is square for 2D DOA estimation. However the variance is not evident, only $0.01^{\circ}$. Moreover we see from the equation (28) that according to the CRLB analytical expression of the particular array configurations the obtained results are rather small for one signal case. Moreover, the error estimation distribution is uniform because the arrays are symmetrical. 
All the arrays do not dependent on one signal azimuth location, because the shape of the antenna element arrangements is symmetric. In the case of one signal RMSE of estimates of MUSIC via the circular array is a bit less than the others have. That matches CRB results (27-28). The accuracy of DOA-estimation in azimuth-elevation scenario is highly determined by the source elevation angle. The best accuracy can be reached if the signal source is close to $\varphi=45^{\circ}$.

\section{EXACT EQUATION FOR CRAMER-RAO LOWER BOUND FOR DOA VIA NON-SYMMETRIC ARRAYS}

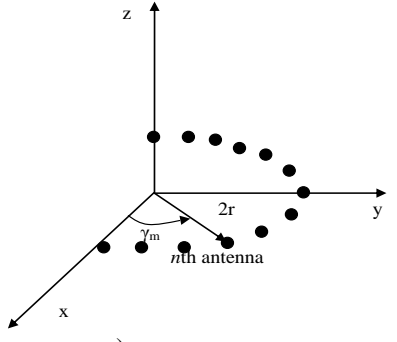

a)

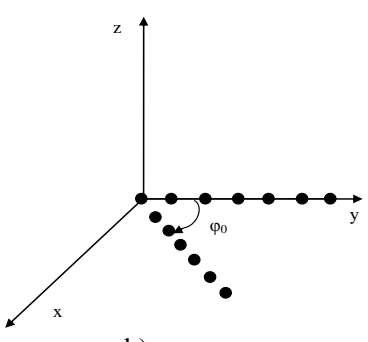

b)
Fig. 4. Schematic of a) Arc- and b) L-shaped arrays.

It is known that L-shaped arrays (fig. $4 \mathrm{~b}$ ), as well as arcshaped ones (Fig. 4a) [18, 26] may give better performances of DOA-estimation, than circular arrays (Fig. 2a) for one signal source case. Therefore the problem of choosing the best antenna array geometry is very important for multiple signal sources as well as a radio direction finding method.

The considered antenna arrays depicted in Fig. 4 consist of 24 omnidirectional elements, the interelement spacing is halfwavelength, angle between two sides of the L-shaped array is equal to $\varphi_{0}=45^{\circ}$, the radius of the arc-shaped array in comparison with the standard circular one is doubled while using the expression (6). We will use the following steering vector equation for the L-shaped antenna array [9]:

$$
a_{n}^{L}(\phi, \theta)=\left\{\begin{array}{c}
e^{j n k_{x} d_{x}}, n=0 \div \frac{N}{2}-1 \\
e^{j(n-\mathrm{N} / 2) k d_{x} \sin \phi \cos \left(\theta-\varphi_{0}\right)}, n=\frac{N}{2} \div N-1
\end{array}\right.
$$

Consider a single source scenario, as in the example above.

From Fig. 5 it is seen that the best antenna array for DOAestimation in the azimuth and elevation is the arc-shaped geometry.

Let us obtain the exact equations of the $A R$ term for calculating CRLB via the considered non-symmetric antenna arrays and compare them with the expression for the circular AR (25-26). Then, after simplifying the expression (18) for the arc-shaped array the $A R$ term can be written as:

$$
\begin{gathered}
A R_{\theta}^{\operatorname{arc}=} 702\left(\frac{\lambda}{2} \sin \theta \sin \varphi\right)^{2}+135\left(\frac{\lambda}{2} \cos \theta \sin \varphi\right)^{2}- \\
76\left(\frac{\lambda}{2} \sin \varphi\right)^{2} \sin \theta \cos \theta
\end{gathered}
$$

$$
\begin{gathered}
A R_{\varphi}^{\text {arc }}=702\left(\frac{\lambda}{2} \cos \theta \cos \varphi\right)^{2}+135\left(\frac{\lambda}{2} \sin \theta \cos \varphi\right)^{2}- \\
76\left(\frac{\lambda}{2} \cos \varphi\right)^{2} \cos \theta \sin \theta
\end{gathered}
$$

The equation (18) for the L-shaped array becomes:

$$
\begin{gathered}
A R_{\theta}^{L}=219\left(\frac{\lambda}{2} \sin \theta \sin \varphi\right)^{2}+198\left(\frac{\lambda}{2} \cos \theta \sin \varphi\right)^{2}+ \\
93\left(\frac{\lambda}{2} \sin \varphi\right)^{2} \sin \theta \cos \theta \\
A R_{\varphi}^{L}=219\left(\frac{\lambda}{2} \cos \theta \cos \varphi\right)^{2}+198\left(\frac{\lambda}{2} \sin \theta \cos \varphi\right)^{2}+ \\
93\left(\frac{\lambda}{2} \cos \varphi\right)^{2} \cos \theta \sin \theta
\end{gathered}
$$

As it can be seen from the expressions (30-33), the variable $A R$ for the arc-shaped array is bigger than others. Wherein:

$$
\begin{gathered}
A R_{\varphi, \theta}^{a r c}>A R_{\varphi, \theta}^{c i r c} \\
C R B_{\varphi, \theta}^{a r c}<C R B_{\varphi, \theta}^{\text {circ }}
\end{gathered}
$$

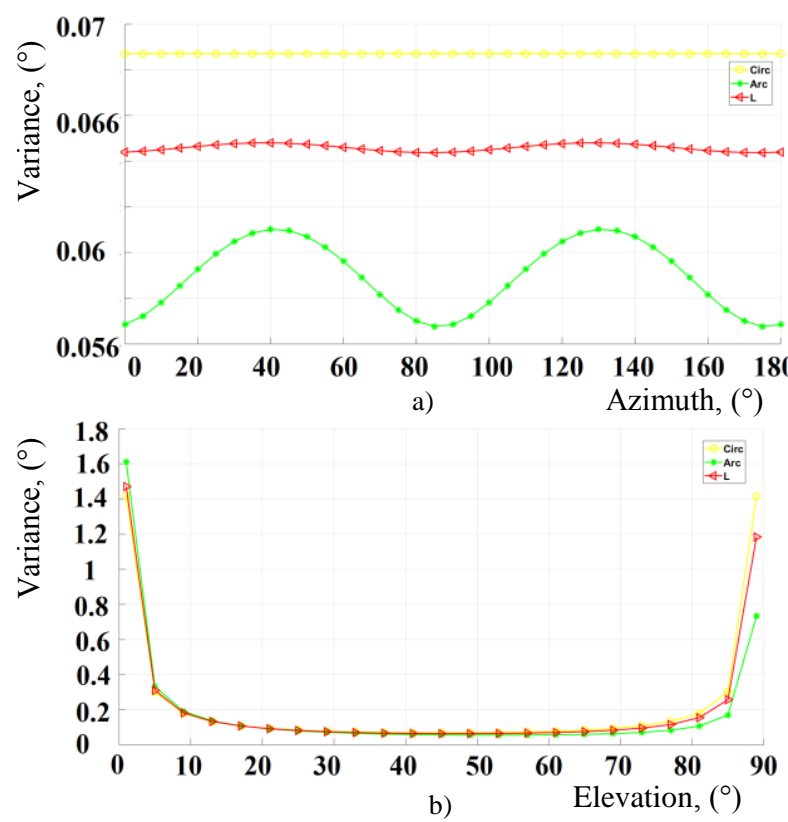

Fig. 5. Cramer-Rao bound a) $\theta=0^{\circ}-180^{\circ}, \varphi=45^{\circ}$, b) $\theta=1^{\circ}, \varphi=1^{\circ}-89^{\circ}$ for one signal case.

Then it turns out that the DOA-estimation accuracy via the arc-shaped arrays is better than the standard circular array. However, there is some hopping in the azimuth scanning plane. Although for the entire scanning range there are less the errors than via the circular one.

In other words it is found out that the super-resolution methods like MUSIC should be used via arc-shaped antenna 
arrays in order to obtain more accurate estimates of twodimensional angular coordinates of a signal source including the worst case source locations, i.e. away from the middle of the elevation angle. This case is produced because this kind of arrays posses dimensions two times the circular ones with the same number of elements.

\section{GENERAL EXPRESSION OF CRLB FOR ARRAYS WITH OMNIDIRECTIONAL ELEMENTS (SINGLE SIGNAL-TWO ELEMENTS)}

As it was already shown from the expressions (15-18), accuracy of direction finding in azimuth and elevation is mainly determined by the antenna array geometry, namely the coefficient $A R$ (18). Consider it more in detail. Let us assume that there is an array consisting of two elements and single signal source. Then the following expression is derived in Appendix A:

$$
\begin{gathered}
A R_{2}= \\
\left(x_{1} k_{x}^{\prime}+y_{1} k_{y}^{\prime}+z_{1} k_{z}^{\prime}\right) . \\
\left(\begin{array}{l}
k_{x}^{\prime}\left(1 / 2 x_{1}-1 / 2 x_{2}\right)+ \\
k_{y}^{\prime}\left(1 / 2 y_{1}-1 / 2 y_{2}\right)+k_{z}^{\prime}\left(1 / 2 z_{1}-1 / 2 z_{2}\right)
\end{array}\right)+ \\
\left(x_{2} k_{x}^{\prime}+y_{2} k_{y}^{\prime}+z_{2} k_{z}^{\prime}\right) . \\
\left(\begin{array}{l}
k_{x}^{\prime}\left(-1 / 2 x_{1}+1 / 2 x_{2}\right)+ \\
k_{y}^{\prime}\left(-1 / 2 y_{1}+1 / 2 y_{2}\right)+k_{z}^{\prime}\left(-1 / 2 z_{1}+1 / 2 z_{2}\right)
\end{array}\right)
\end{gathered}
$$

Accept that $x_{i} k_{x}^{\prime}=d x_{i}, y_{i} k_{y}^{\prime}=d y_{i}, z_{i} k_{z}^{\prime}=d z_{i}$, and also that the considered antennas are planar, i.e. $z_{i}=0$. Then it turns out that:

$$
\begin{aligned}
& A R_{2}=\left(d x_{1}+d y_{1}\right)\left(\left(1 / 2 d x_{1}-1 / 2 d x_{2}\right)+\left(1 / 2 d y_{1}-1 / 2 d y_{2}\right)\right)+ \\
& \left(d x_{2}+d y_{2}\right)\left(\left(-1 / 2 d x_{1}+1 / 2 d x_{2}\right)+\left(-1 / 2 d y_{1}+1 / 2 d y_{2}\right)\right)
\end{aligned}
$$

We open parentheses in the formula (37) and get:

$$
\begin{gathered}
A R_{2}=1 / 2\left(d x_{1}-d x_{2}\right)^{2}+1 / 2\left(d y_{1}-d y_{2}\right)^{2} \\
+\left(d x_{1}-d x_{2}\right)\left(d y_{1}-d y_{2}\right)
\end{gathered}
$$

It can be seen from the last expression (38) that the CramerRao lower bound of the planar arrays mainly determined by the sum of squares of the difference of coordinates along the $x$ and $y$ axes. In other words, increasing the difference between the elements quadratically reduces errors of the DOAestimations on the azimuth or elevation planes.

Consider a single signal source scenario, whose coordinate in azimuth is $\theta=70^{\circ}$. Let us take the standard circular twoelement antenna array (Fig. 6a), as well as the array constructed by maximizing the last expression (38). The radius is $r=0.0442 \mathrm{~m}$. After the maximization (38), the coordinates of the elements are $\left(x_{1}=0.0415 ; y_{1}=-0.0151\right),\left(x_{2}=-0.0415\right.$; $\left.y_{2}=0.0151\right)$, as shown in Fig. $6 \mathrm{~b}$.

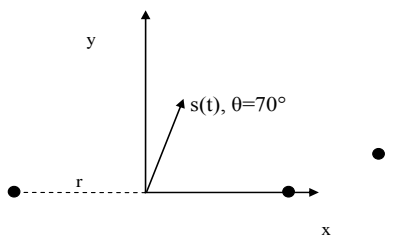

a)

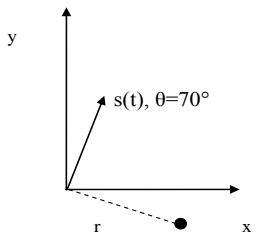

b)
Fig. 6. Schematic of a) standard circular, b) optimized arrays

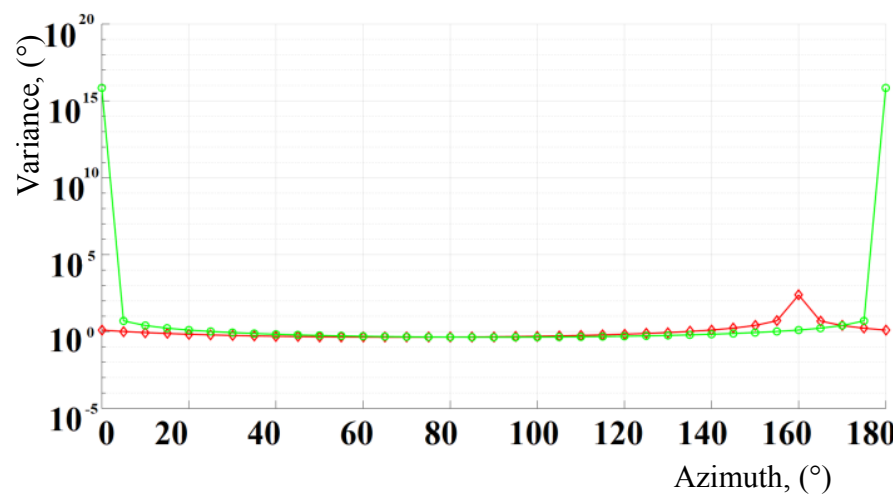

Fig. 7. CRLB of the antenna arrays: the green curve relates to the usual array from Fig. 5a, the red curve relates to the optimized array from Fig. 5b.

The results of the accuracy calculation in azimuth of the two antenna arrays are shown in Fig. 7. After viewing the obtained curves we can say that there is no gain after rotating the array by $20^{\circ}$ clockwise.

In addition, in this case the term $A R$ of the usual array depicted in Fig. 6a is equal to:

$$
\begin{gathered}
A R_{\theta}^{\text {circ }}=4\left(\frac{\lambda}{2} \sin \theta \sin \varphi\right)^{2} \\
A R_{\theta}^{\text {circ }}\left(\theta=70^{\circ}, \varphi=45^{\circ}\right)=0.0017,
\end{gathered}
$$

but for the optimized one from Fig. $7 \mathrm{~b}$ on the azimuth $70^{\circ}$ :

$$
\begin{gathered}
A R_{\theta}^{\text {optim }}=3.5272\left(\frac{\lambda}{2} \sin \theta \sin \varphi\right)^{2}- \\
2.5668\left(\frac{\lambda}{2} \sin \theta \sin \varphi\right)\left(\frac{\lambda}{2} \cos \theta \sin \varphi\right)+0.4670\left(\frac{\lambda}{2} \cos \theta \sin \varphi\right)^{2} \\
A R_{\theta}^{\text {optim }}\left(\theta=70^{\circ}, \varphi=45^{\circ}\right)=0.0011
\end{gathered}
$$

Obviously, there is no difference between these antenna arrays.

\section{GENERAL EXPRESSION OF CRLB FOR ARRAYS WITH OMNIDIRECTIONAL ELEMENTS (SINGLE SIGNAL-THREE ELEMENTS)}

Assume that there is a planar antenna array consisting of three elements in the $X Y$ plane and single signal source having arbitrary coordinates $\theta, \varphi$. Based on (18) we can write down the following formula (the proof is in Appendix B): 


$$
\begin{gathered}
A R_{3}= \\
=\left(d x_{1}+d y_{1}\right)\left(\begin{array}{l}
\left(2 / 3 d x_{1}-1 / 3 d x_{2}-1 / 3 d x_{3}\right)+ \\
\left(2 / 3 d y_{1}-1 / 3 d y_{2}-1 / 3 d y_{3}\right)
\end{array}\right)+ \\
\left(d x_{2}+d y_{2}\right)\left(\begin{array}{l}
\left(-1 / 3 d x_{1}+2 / 3 d x_{2}-1 / 3 d x_{3}\right)+ \\
\left(-1 / 3 d y_{1}+2 / 3 d y_{2}-1 / 3 d y_{3}\right)
\end{array}\right)+ \\
\left(d x_{3}+d y_{3}\right)\left(\begin{array}{l}
\left(-1 / 3 d x_{1}-1 / 3 d x_{2}+2 / 3 d x_{3}\right)+ \\
\left(-1 / 3 d y_{1}-1 / 3 d y_{2}+2 / 3 d y_{3}\right)
\end{array}\right)
\end{gathered}
$$

Then it is easy to simplify the last expression: $A R_{3}=\frac{1}{3}\left[\begin{array}{l}\left(d x_{1}-d x_{2}\right)^{2}+\left(d x_{1}-d x_{3}\right)^{2}+\left(d x_{3}-d x_{2}\right)^{2}+ \\ \left(d y_{1}-d y_{2}\right)^{2}+\left(d y_{1}-d y_{3}\right)^{2}+\left(d y_{3}-d y_{2}\right)^{2}\end{array}\right]+$ $\frac{1}{3}\left[\begin{array}{l}4 d y_{1} d x_{1}-2 d y_{2} d x_{1}-2 d y_{3} d x_{1}-2 d x_{2} d y_{1}-2 d x_{3} d y_{1}+ \\ 4 d y_{2} d x_{2}-2 d y_{3} d x_{2}-2 d x_{3} d y_{2}+4 d y_{3} d x_{3}\end{array}\right]=$ $\frac{1}{3}\left[\begin{array}{l}\left(d x_{1}-d x_{2}\right)^{2}+\left(d x_{1}-d x_{3}\right)^{2}+\left(d x_{3}-d x_{2}\right)^{2}+ \\ \left(d y_{1}-d y_{2}\right)^{2}+\left(d y_{1}-d y_{3}\right)^{2}+\left(d y_{3}-d y_{2}\right)^{2}\end{array}\right]+$

$$
2 / 3\left[\begin{array}{l}
\left(2 d x_{1}-d x_{2}-d x_{3}\right)\left(2 d y_{1}-d y_{2}-d y_{3}\right)+ \\
\left(-d x_{1}+2 d x_{2}-d x_{3}\right)\left(-d y_{1}+2 d y_{2}-d y_{3}\right)+ \\
\left(-d x_{1}-d x_{2}+2 d x_{3}\right)\left(-d y_{1}-d y_{2}+2 d y_{3}\right)
\end{array}\right]
$$

From the formula (42) it is seen that the accuracy of direction-of-arrival estimation by planar antenna arrays of three non-directional elements is determined by the sum of the squares of the differences of their coordinates as well.

Consider the same scenario like almost above, i.e. the single signal source has the azimuth coordinate $\theta=50^{\circ}$ and elevation $\varphi=45^{\circ}$. Also we will use the common three-element circular antenna array depicted in Fig. 8a, and additionally an array which has been constructed by maximizing (42). The radius is $r=0.0442 \mathrm{~m}$. After maximization (42), the coordinates of the elements are $\left(x_{1}=-0.0328 ; y_{1}=0.0296\right),\left(x_{2}=-0.0349 ; y_{2}=\right.$ $0.0272),\left(x_{3}=0.0339 ; y_{3}=-0.0284\right)$, as shown in Fig. 8b.

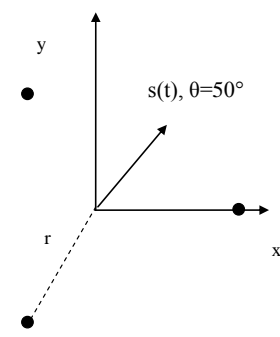

a)

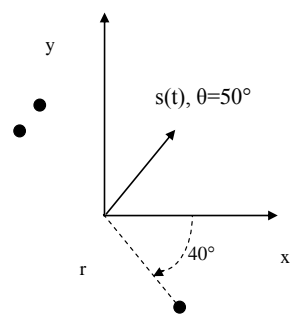

b)
Fig. 8. Schematic of a) standard circular, b) optimized arrays

In addition, in this case the term $A R$ of the standard circular array is equal to:

$$
\begin{aligned}
& A R_{\theta}^{\text {circ }}=3\left(\frac{\lambda}{2} \sin \theta \sin \varphi\right)^{2}+3\left(\frac{\lambda}{2} \cos \theta \sin \varphi\right)^{2}, \\
& A R_{\theta}^{\text {circ }}\left(\theta=50^{\circ}, \varphi=45^{\circ}\right)=0.00146
\end{aligned}
$$

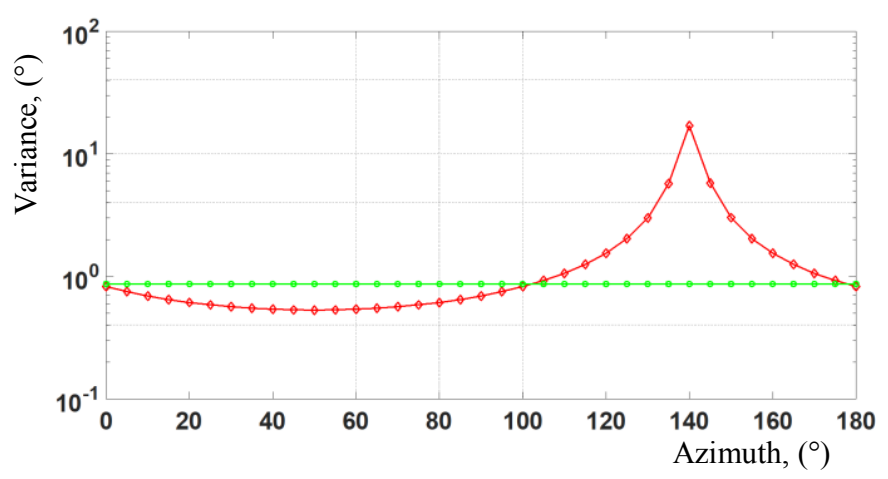

Fig. 9. CRLB of the antenna arrays: the green curve relates to the usual circular array from Fig. 8a, the red curve relates to the optimized array from Fig. 8b.

but consequently for the optimized on the azimuth $70^{\circ}$ it is:

$$
\begin{gathered}
A R_{\theta}^{\text {opt }}=4.7\left(\frac{\lambda}{2} \sin \theta \sin \varphi\right)^{2}- \\
3.4\left(\frac{\lambda}{2} \sin \varphi\right)^{2}(\sin \theta \cos \theta)+0.6\left(\frac{\lambda}{2} \cos \theta \sin \varphi\right)^{2} \\
A R_{\theta}^{\text {optim }}\left(\theta=50^{0}, \varphi=45^{\circ}\right)=0.00153
\end{gathered}
$$

From the graphs in Fig. 9 and the values obtained in (43-44) it is seen that a new form of antenna array provides a reduction of errors in the range $\theta=15^{\circ}-95^{\circ}$.

\section{GENERAL EXPRESSION OF CRLB FOR ARRAYS WITH OMNIDIRECTIONAL ELEMENTS (SINGLE SigNAL-SEVERAL ELEMENTS)}

We are now going to derive an expression for a single source and an arbitrary number of non-directional antenna elements of planar antenna arrays (Appendix C) based on (18).

$$
\begin{gathered}
A R_{n}=\left(d x_{1}+d y_{1}\right)\left(\begin{array}{l}
\left(\frac{N-1}{N} d x_{1}-\frac{1}{N} d x_{2} \ldots-\frac{1}{N} d x_{N}\right)+ \\
\left(\frac{N-1}{N} d y_{1}-\frac{1}{N} d y_{2} \ldots-\frac{1}{N} d y_{N}\right)
\end{array}\right)+ \\
\left(d x_{2}+d y_{2}\right)\left(\begin{array}{l}
\left(-\frac{1}{N} x_{1}+\frac{N-1}{N} d x_{2} \ldots-\frac{1}{N} d x_{N}\right)+ \\
\left(-\frac{1}{N} d y_{1}+\frac{N-1}{N} d y_{2}-\frac{1}{N} d y_{N}\right)
\end{array}\right)+\ldots \\
\left(d x_{N}+d y_{N}\right)\left(\begin{array}{l}
\left(-\frac{1}{N} d x_{1}-\frac{1}{N} d x_{2} \ldots+\frac{N-1}{N} d x_{N}\right)+ \\
\left(-\frac{1}{N} d y_{1}-\frac{1}{N} d y_{2} \ldots+\frac{N-1}{N} d y_{N}\right)
\end{array}\right)
\end{gathered}
$$

After simplifying the expression (45) we obtain:

$$
\begin{gathered}
A R=\frac{1}{N} \sum_{i=1}^{N} \sum_{j=1}^{N}\left[\left(d x_{i}-d x_{j}\right)^{2}+\left(d y_{i}-d y_{j}\right)^{2}\right]+ \\
\frac{N-1}{N} \sum_{n=1}^{N}\left[\left((N-1) d x_{n}-d x_{i \neq n} \ldots-d x_{j \neq n}\right) \cdot\right] \\
\left.\left((N-1) d y_{n}-d y_{i \neq n} \ldots-d y_{j \neq n}\right)\right]
\end{gathered}
$$


Also let us consider the same scenario like above, i.e. the single signal source has the azimuth coordinate $\theta=120^{\circ}$. Also we will use the usual six-element circular antenna array depicted in Fig. 10a, and additionally an array which has been constructed by maximizing the last expression. In this case the radius is $r=2 * 0.0442 \mathrm{~m}$. After maximization, the coordinates of the elements are $\left(x_{1}=0.0632 ; y_{1}=0.0592\right),\left(x_{2}=0.0811 ; y_{2}=\right.$ $0.0329),\left(x_{3}=-0.0735 ; y_{3}=-0.0466\right),\left(x_{4}=-0.0632 ; y_{4}=-\right.$ $0.0592),\left(x_{5}=-0.0811 ; y_{5}=-0.0329\right),\left(x_{6}=0.0735 ; y_{6}=0.0466\right)$ as shown in Fig. $10 \mathrm{~b}$.

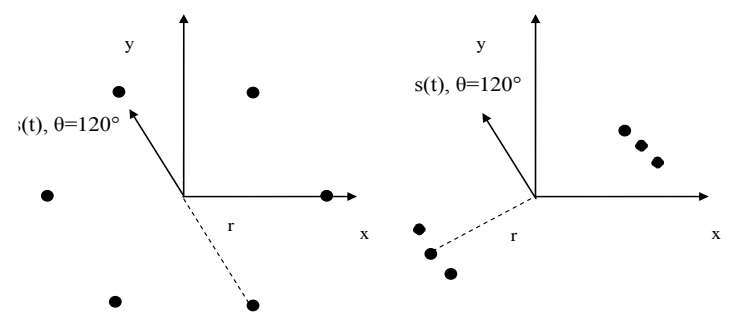

a)

b)

Fig. 10. Schematic of a) standard circular, b) optimized arrays

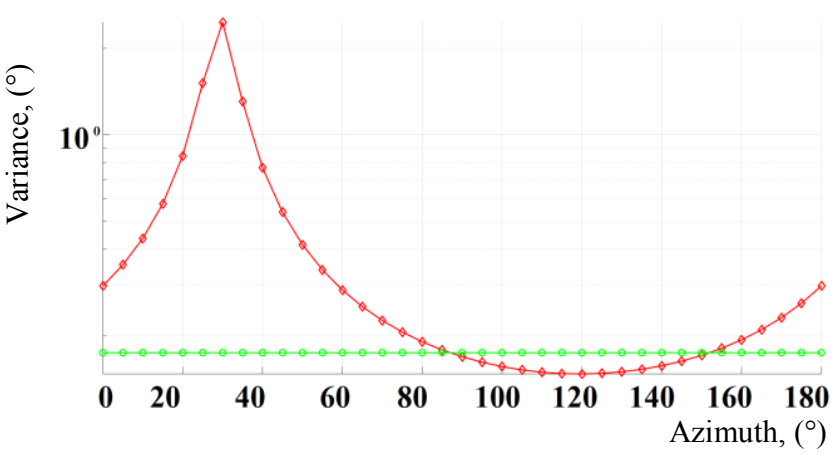

Fig. 11. CRLB of the antenna arrays: the green curve relates to the usual array from Fig. 10a, the red curve relates to the optimized array from Fig. 10b.

In addition, in this case the term $A R$ of the common circular array (Fig. 10a) is equal to:

$$
\begin{gathered}
A R_{\theta}^{\text {circ }}=6\left(\frac{\lambda}{2} \sin \theta \sin \varphi\right)^{2}+6\left(\frac{\lambda}{2} \cos \theta \sin \varphi\right)^{2}, \\
A R_{\theta}^{\text {circ }}\left(\theta=120^{\circ}, \varphi=45^{\circ}\right)=0.0117
\end{gathered}
$$

For the optimized array (Fig. 10b) on the azimuth $120^{\circ}$ it is:

$$
\begin{gathered}
A R_{\theta}^{o p t}=8.2\left(\frac{\lambda}{2} \sin \theta \sin \varphi\right)^{2}- \\
10\left(\frac{\lambda}{2} \sin \varphi\right)^{2}(\sin \theta \cos \theta)+3.5\left(\frac{\lambda}{2} \cos \theta \sin \varphi\right)^{2} \\
A R_{\theta}^{\text {optim }}\left(\theta=120^{\circ}, \varphi=45^{\circ}\right)=0.0222
\end{gathered}
$$

This case with azimuth source location of $\theta=120^{\circ}$ is taken in order to show the diversity of the applied method. From the curves depicted in Fig. 11 it is seen that the obtained antenna array (Fig. 10b) after optimizing the expression (46) provides a reduction in the direction-finding errors both around azimuth $120^{\circ}$ and wide around it. This property can be used for implementation of the digital adaptive antennas with sector switching.

Moreover, after viewing the shape of the modified forms of antenna arrays from Fig. 6, 8, 10 we can make some generalizations regarding the construction of the optimal shape of a planar antenna array. Namely, to scan a signal inside a specific area it is necessary to build two parallel antenna arrays, turned around by the appropriate angle. This angle will approximately correspond to the area of the intended scan. This technique and recommendations for the construction of planar antenna arrays can be applied to the cases in paragraphs IV-V.

Remind that the single signal source has the azimuth coordinate $\theta=70^{\circ}$ and 24-element circular antenna array depicted in Fig. 12a is used, and additionally an array which has been constructed by maximizing (46). The radius is $r=$ $0.2392 \mathrm{~m}$. The constructed array is depicted in Fig. $12 \mathrm{~b}$.
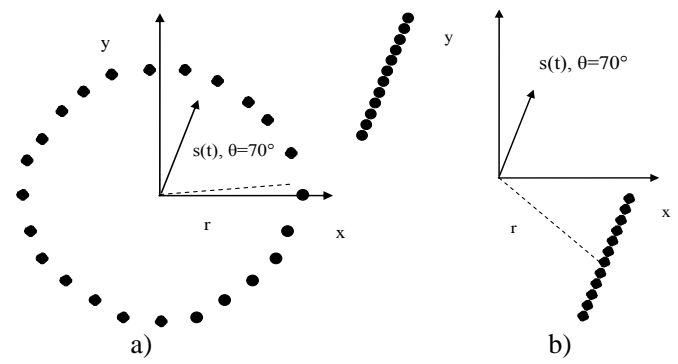

Fig. 12. Schematic of a) standard circular, b) optimized arrays

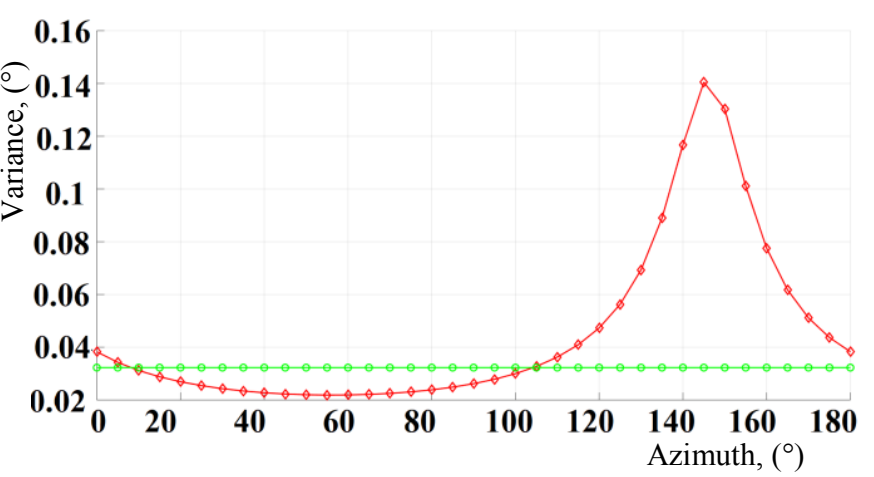

Fig. 13. CRLB of the antenna arrays: the green curve relates to the usual array from Fig. 12a, the red curve relates to the optimized array from Fig. 12b.

$$
\begin{gathered}
A R_{\theta}^{\text {circ }}=175\left(\frac{\lambda}{2} \sin \theta \sin \varphi\right)^{2}+175\left(\frac{\lambda}{2} \cos \theta \sin \varphi\right)^{2} \\
A R_{\theta}^{\text {circ }}\left(\theta=70^{\circ}, \varphi=45^{\circ}\right)=0.3413 \\
A R_{\theta}^{\text {opt }}=302\left(\frac{\lambda}{2} \sin \theta \sin \varphi\right)^{2}- \\
391\left(\frac{\lambda}{2} \sin \varphi\right)^{2}(\sin \theta \cos \theta)+141\left(\frac{\lambda}{2} \cos \theta \sin \varphi\right)^{2} \\
A R_{\theta}^{\text {optim }}\left(\theta=70^{\circ}, \varphi=45^{\circ}\right)=0.7974
\end{gathered}
$$


From expressions (49-50), as well as graphs in Fig. 13 it is seen that thanks to the new shape of the antenna array the errors within the target sector are reduced.

\section{CONCLUSION}

The adaptation of the Cramer-Rao lower bound expression for the 3D direction-finding tasks of planar antenna arrays with omnidirectional emitters is carried out. The influence of the azimuth and elevation coordinates of radio emission sources on the accuracy of radio direction finding is estimated. Also the exact expressions for estimation of the accuracy of direction-of-arrival using the Cramer-Rao lower bound via planar antenna arrays of the symmetric shape has obtained. Moreover the equations are legal for the particular case of one receiving signal. It is proved by math expressions that the circular antenna arrays are the best for DOA estimation with one receive source among the arrays of the symmetrical shape. If an arbitrary geometry is considered like L- or arc- shaped arrays then the non-symmetrical kind of antennas can be used to improve the direction-of-arrival estimation accuracy.

In addition, the exact general expressions of the CramerRao lower bound for planar antenna arrays were obtained. The formulas describe the dependence of the direction finding accuracy in azimuth or elevation angle from the positions of antenna elements. It is found out that the pattern is quadratic. The obtained formulas allow the antenna elements to be positioned so that the direction-of-arrival errors will be significantly reduced in the target scanning sector, exceeding the circular antenna arrays by this measurement.

The results for the optimized arrays have better performance only on specified ranges of azimuth values. This is due to the fact that minimization of the CRLB for a certain azimuth value is fulfilled. These results can be used to build a system with switched elements to scan a specific area of space with reduced direction finding error.

\section{APPENDIX}

A. Proof of (36)

$$
\begin{aligned}
& A R_{2}=\left[\begin{array}{ll}
\left(\mathbf{k}_{1}^{\prime} \mathbf{r}_{1}\right)^{H} \mathbf{k}^{\prime} & \left(\mathbf{k}^{\prime} \mathbf{r}_{2}\right)^{H} \mathbf{k}^{\prime}
\end{array}\right] \cdot\left[\begin{array}{cc}
1 / 2 & -1 / 2 \\
-1 / 2 & 1 / 2
\end{array}\right]\left[\begin{array}{l}
\mathbf{r}_{1} \\
\mathbf{r}_{2}
\end{array}\right]= \\
& \left.\left[\left(\mathbf{k}_{1}^{\prime}\right)^{H}\right)^{\prime} \mathbf{k}^{\prime} \quad\left(\mathbf{k}^{\prime} \mathbf{r}_{2}\right)^{H} \mathbf{k}^{\prime}\right] \cdot\left[\begin{array}{c}
1 / 2 \mathbf{r}_{1}-1 / 2 \mathbf{r}_{2} \\
-1 / 2 \mathbf{r}_{1}+1 / 2 \mathbf{r}_{2}
\end{array}\right]= \\
& \left(\mathbf{k}^{\prime} \mathbf{r}_{1}\right)^{H} \mathbf{k}^{\prime}\left(1 / 2 \mathbf{r}_{1}-1 / 2 \mathbf{r}_{2}\right)+\left(\mathbf{k}_{2} \mathbf{r}^{H}\right)^{H} \mathbf{k}^{\prime}\left(-1 / 2 \mathbf{r}_{1}+1 / 2 \mathbf{r}_{2}\right)= \\
& \left([ k _ { x } ^ { \prime } , k _ { y } ^ { \prime } , k _ { z } ^ { \prime } [ \begin{array} { l } 
{ x _ { 1 } } \\
{ y _ { 1 } } \\
{ z _ { 1 } }
\end{array} ] ) ^ { H } \left[k_{x}^{\prime}, k_{y}^{\prime}, k_{z}^{\prime}\left(1 / 2\left[\begin{array}{l}
x_{1} \\
y_{1} \\
z_{1}
\end{array}\right]-1 / 2\left[\begin{array}{c}
x_{2} \\
y_{2} \\
z_{2}
\end{array}\right]\right)+\right.\right. \\
& \left(\left[k_{x}^{\prime}, k_{y}^{\prime}, k_{z}^{\prime}\left[\begin{array}{l}
x_{2} \\
y_{2} \\
z_{2}
\end{array}\right]\right)^{H}\left[k_{x}^{\prime}, k_{y}^{\prime}, k_{z}^{\prime}\right]-1 / 2\left[\begin{array}{l}
x_{1} \\
y_{1} \\
z_{1}
\end{array}\right]+1 / 2\left[\begin{array}{c}
x_{2} \\
y_{2} \\
z_{2}
\end{array}\right]\right)= \\
& \left(x_{1} k_{x}^{\prime}+y_{1} k_{y}^{\prime}+z_{1} k_{z}^{\prime}\right) . \\
& \left(\begin{array}{l}
k_{x}^{\prime}\left(1 / 2 x_{1}-1 / 2 x_{2}\right)+ \\
k_{y}^{\prime}\left(1 / 2 y_{1}-1 / 2 y_{2}\right)+k_{z}^{\prime}\left(1 / 2 z_{1}-1 / 2 z_{2}\right)
\end{array}\right)+ \\
& \left(x_{2} k_{x}^{\prime}+y_{2} k_{y}^{\prime}+z_{2} k_{z}^{\prime}\right) \text {. } \\
& \left(k_{x}^{\prime}\left(-1 / 2 x_{1}+1 / 2 x_{2}\right)+\right. \\
& \left(k_{y}^{\prime}\left(-1 / 2 y_{1}+1 / 2 y_{2}\right)+k_{z}^{\prime}\left(-1 / 2 z_{1}+1 / 2 z_{2}\right)\right)
\end{aligned}
$$

Then the equation (A.1) can be simplified:

$$
\begin{gathered}
A R_{2}=d x_{1}\left(1 / 2 d x_{1}-1 / 2 d x_{2}\right)+d x_{1}\left(1 / 2 d y_{1}-1 / 2 d y_{2}\right)+ \\
d y_{1}\left(1 / 2 d x_{1}-1 / 2 d x_{2}\right)+d y_{1}\left(1 / 2 d y_{1}-1 / 2 d y_{2}\right)+ \\
d x_{2}\left(-1 / 2 d x_{1}+1 / 2 d x_{2}\right)+d x_{2}\left(-1 / 2 d y_{1}+1 / 2 d y_{2}\right)+ \\
d y_{2}\left(-1 / 2 d x_{1}+1 / 2 d x_{2}\right)+d y_{2}\left(-1 / 2 d y_{1}+1 / 2 d y_{2}\right)= \\
1 / 2 d x_{1}^{2}-1 / 2 d x_{2} d x_{1}+1 / 2 d y_{1} d x_{1}-1 / 2 d y_{2} d x_{1}+ \\
1 / 2 d x_{1} d y_{1}-1 / 2 d x_{2} d y_{1}+1 / 2 d y_{1}^{2}-1 / 2 d y_{2} d y_{1}+ \\
-1 / 2 d x_{1} d x_{2}+1 / 2 d x_{2}^{2}-1 / 2 d y_{1} d x_{2}+1 / 2 d y_{2} d x_{2}- \\
1 / 2 d x_{1} d y_{2}+1 / 2 d x_{2} d y_{2}-1 / 2 d y_{1} d y_{2}+1 / 2 d y_{2}^{2}= \\
\left(1 / 2 d x_{1}^{2}-d x_{1} d x_{2}+1 / 2 d x_{2}^{2}\right)+ \\
\left(1 / 2 d y_{1}^{2}-d y_{1} d y_{2}+1 / 2 d y_{2}^{2}\right)+ \\
\left(d y_{1} d x_{1}+d y_{2} d x_{2}-d y_{2} d x_{1}-d y_{1} d x_{2}\right)= \\
1 / 2\left(d x_{1}-d x_{2}\right)^{2}+1 / 2\left(d y_{1}-d y_{2}\right)^{2}+\left(d x_{1}-d x_{2}\right)\left(d y_{1}-d y_{2}\right)
\end{gathered}
$$

B. Proof of (41)

$$
\begin{aligned}
& A R_{3}=\left[\begin{array}{lll}
\left(\mathbf{k}^{\prime} \mathbf{r}_{1}\right)^{H} \mathbf{k}^{\prime} & \left(\mathbf{k}^{\prime} \mathbf{r}_{2}\right)^{H} \mathbf{k}^{\prime} \quad\left(\mathbf{k}^{\prime} \mathbf{r}_{3}\right)^{H} \mathbf{k}^{\prime}
\end{array}\right] . \\
& {\left[\begin{array}{ccc}
2 / 3 & -1 / 3 & -1 / 3 \\
-1 / 3 & 2 / 3 & -1 / 3 \\
-1 / 3 & -1 / 3 & 2 / 3
\end{array}\right]\left[\begin{array}{l}
\mathbf{r}_{1} \\
\mathbf{r}_{2} \\
\mathbf{r}_{3}
\end{array}\right]=} \\
& \left([ k _ { x } ^ { \prime } , k _ { y } ^ { \prime } , k _ { z } ^ { \prime } [ \begin{array} { l } 
{ x _ { 1 } } \\
{ y _ { 1 } } \\
{ z _ { 1 } }
\end{array} ] ) ^ { H } \left[k_{x}^{\prime}, k_{y}^{\prime}, k_{z}^{\prime}\left\{2 / 3\left[\begin{array}{l}
x_{1} \\
y_{1} \\
z_{1}
\end{array}\right]-1 / 3\left[\begin{array}{c}
x_{2} \\
y_{2} \\
z_{2}
\end{array}\right]-1 / 3\left[\begin{array}{l}
x_{3} \\
y_{2} \\
z_{3}
\end{array}\right]\right)+\right.\right. \\
& \left(\left[k_{x}^{\prime}, k_{y}^{\prime}, k_{z}^{\prime}\left[\begin{array}{l}
x_{2} \\
y_{2} \\
z_{2}
\end{array}\right]\right)^{H}\left[k_{x}^{\prime}, k_{y}^{\prime}, k_{z}^{\prime}\right]-1 / 3\left[\begin{array}{l}
x_{1} \\
y_{1} \\
z_{1}
\end{array}\right]+2 / 3\left[\begin{array}{c}
x_{2} \\
y_{2} \\
z_{2}
\end{array}\right]-1 / 3\left[\begin{array}{l}
x_{3} \\
y_{2} \\
z_{3}
\end{array}\right]\right)+ \\
& \left(\left[k_{x}^{\prime}, k_{y}^{\prime}, k_{z}^{\prime}\left[\begin{array}{l}
x_{3} \\
y_{3} \\
z_{3}
\end{array}\right]\right)^{H}\left[k_{x}^{\prime}, k_{y}^{\prime}, k_{z}^{\prime}\right\}-1 / 3\left[\begin{array}{l}
x_{1} \\
y_{1} \\
z_{1}
\end{array}\right]-1 / 3\left[\begin{array}{l}
x_{2} \\
y_{2} \\
z_{2}
\end{array}\right]+2 / 3\left[\begin{array}{l}
x_{3} \\
y_{2} \\
z_{3}
\end{array}\right]\right) \\
& =\left(d x_{1}+d y_{1}\right)\left(\begin{array}{l}
\left(2 / 3 d x_{1}-1 / 3 d x_{2}-1 / 3 d x_{3}\right)+ \\
\left(2 / 3 d y_{1}-1 / 3 d y_{2}-1 / 3 d y_{3}\right)
\end{array}\right)+ \\
& \left(d x_{2}+d y_{2}\right)\left(\begin{array}{l}
\left(-1 / 3 d x_{1}+2 / 3 d x_{2}-1 / 3 d x_{3}\right)+ \\
\left(-1 / 3 d y_{1}+2 / 3 d y_{2}-1 / 3 d y_{3}\right)
\end{array}\right)+ \\
& \left(d x_{3}+d y_{3}\right)\left(\begin{array}{l}
\left(-1 / 3 d x_{1}-1 / 3 d x_{2}+2 / 3 d x_{3}\right)+ \\
\left(-1 / 3 d y_{1}-1 / 3 d y_{2}+2 / 3 d y_{3}\right)
\end{array}\right)
\end{aligned}
$$

We would like to cancel the parentheses in (B.1) and obtain:

$$
\begin{gathered}
A R_{3}=2 / 3 d x_{1}^{2}-1 / 3 d x_{2} d x_{1}-1 / 3 d x_{3} d x_{1}+2 / 3 d y_{1} d x_{1}- \\
1 / 3 d y_{2} d x_{1}-1 / 3 d y_{3} d x_{1}+2 / 3 d x_{1} d y_{1}-1 / 3 d x_{2} d y_{1}- \\
1 / 3 d x_{3} d y_{1}+2 / 3 d y_{1}^{2}-1 / 3 d y_{2} d y_{1}-1 / 3 d y_{3} d y_{1}+ \\
-1 / 3 d x_{1} d x_{2}+2 / 3 d x_{2}^{2}-1 / 3 d x_{3} d x_{2}+-1 / 3 d y_{1} d x_{2}+ \\
2 / 3 d y_{2} d x_{2}-1 / 3 d y_{3} d x_{2}-1 / 3 d x_{1} d y_{2}+2 / 3 d x_{2} d y_{2}- \\
1 / 3 d x_{3} d y_{2}-1 / 3 d y_{1} d y_{2}+2 / 3 d y_{2}^{2}-1 / 3 d y_{3} d y_{2}+ \\
-1 / 3 d x_{1} d x_{3}-1 / 3 d x_{2} d x_{3}+2 / 3 d x_{3}^{2}+-1 / 3 d y_{1} d x_{3}- \\
1 / 3 d y_{2} d x_{3}+2 / 3 d y_{3} d x_{3}-1 / 3 d x_{1} d y_{3}-1 / 3 d x_{2} d y_{3}+ \\
2 / 3 d x_{3} d y_{3}-1 / 3 d y_{1} d y_{3}-1 / 3 d y_{2} d y_{3}+2 / 3 d y_{3}^{2}
\end{gathered}
$$


We group the terms of the last expression (B.2):

$$
\begin{gathered}
A R_{3}=\left(1 / 3 d x_{1}^{2}-2 / 3 d x_{1} d x_{2}+1 / 3 d x_{2}^{2}\right)+ \\
\left(1 / 3 d x_{1}^{2}-2 / 3 d x_{1} d x_{3}+1 / 3 d x_{3}^{2}\right)+ \\
\left(1 / 3 d x_{3}^{2}-2 / 3 d x_{3} d x_{2}+1 / 3 d x_{2}^{2}\right)_{+} \\
\left(1 / 3 d y_{1}^{2}-2 / 3 d y_{1} d y_{2}+1 / 3 d y_{2}^{2}\right)_{+} \\
\left(1 / 3 d y_{1}^{2}-2 / 3 d y_{3} d y_{1}+1 / 3 d y_{3}^{2}\right)_{+} \\
\left(1 / 3 d y_{2}^{2}-2 / 3 d y_{3} d y_{2}+1 / 3 d y_{3}^{2}\right)_{+} \\
4 / 3 d y_{1} d x_{1}-2 / 3 d y_{2} d x_{1}-2 / 3 d y_{3} d x_{1}- \\
2 / 3 d x_{2} d y_{1}-2 / 3 d x_{3} d y_{1}+4 / 3 d y_{2} d x_{2}- \\
2 / 3 d y_{3} d x_{2}-2 / 3 d x_{3} d y_{2}+4 / 3 d y_{3} d x_{3}
\end{gathered}
$$

Then it is easy to simplify the expression (B.3):

$$
\begin{gathered}
A R_{3}=\frac{1}{3}\left[\begin{array}{l}
\left(d x_{1}-d x_{2}\right)^{2}+\left(d x_{1}-d x_{3}\right)^{2}+\left(d x_{3}-d x_{2}\right)^{2}+ \\
\left(d y_{1}-d y_{2}\right)^{2}+\left(d y_{1}-d y_{3}\right)^{2}+\left(d y_{3}-d y_{2}\right)^{2}
\end{array}\right]+ \\
\frac{1}{3}\left[\begin{array}{l}
4 d y_{1} d x_{1}-2 d y_{2} d x_{1}-2 d y_{3} d x_{1}-2 d x_{2} d y_{1}-2 d x_{3} d y_{1}+ \\
4 d y_{2} d x_{2}-2 d y_{3} d x_{2}-2 d x_{3} d y_{2}+4 d y_{3} d x_{3}
\end{array}\right]= \\
\frac{1}{3}\left[\begin{array}{l}
\left(d x_{1}-d x_{2}\right)^{2}+\left(d x_{1}-d x_{3}\right)^{2}+\left(d x_{3}-d x_{2}\right)^{2}+ \\
\left(d y_{1}-d y_{2}\right)^{2}+\left(d y_{1}-d y_{3}\right)^{2}+\left(d y_{3}-d y_{2}\right)^{2}
\end{array}\right]+ \\
2 / 3\left[\begin{array}{l}
\left(2 d x_{1}-d x_{2}-d x_{3}\right)\left(2 d y_{1}-d y_{2}-d y_{3}\right)+ \\
\left(-d x_{1}+2 d x_{2}-d x_{3}\right)\left(-d y_{1}+2 d y_{2}-d y_{3}\right)+ \\
\left(-d x_{1}-d x_{2}+2 d x_{3}\right)\left(-d y_{1}-d y_{2}+2 d y_{3}\right)
\end{array}\right]
\end{gathered}
$$

\section{Proof of (45)}

$$
\begin{aligned}
& A R_{n}=\left[\begin{array}{llll}
\left(\mathbf{k}^{\prime} \mathbf{r}_{1}\right)^{H} \mathbf{k}^{\prime} & \left(\mathbf{k}_{2}^{\prime} \mathbf{r}_{2}\right)^{H} \mathbf{k}^{\prime} & \cdots & \left(\mathbf{k}^{\prime} \mathbf{r}_{N}\right)^{H} \mathbf{k}^{\prime}
\end{array}\right] . \\
& {\left[\begin{array}{cccc}
\frac{N-1}{N} & -\frac{1}{N} & \ldots & -\frac{1}{N} \\
-\frac{1}{N} & \frac{N-1}{N} & \ldots & -\frac{1}{N} \\
-\frac{1}{N} & -\frac{1}{N} & \ldots & \frac{N-1}{N}
\end{array}\right]\left[\begin{array}{c}
\mathbf{r}_{1} \\
\mathbf{r}_{2} \\
\vdots \\
\mathbf{r}_{N}
\end{array}\right]=} \\
& \left([ k _ { x } ^ { \prime } , k _ { y } ^ { \prime } , k _ { z } ^ { \prime } [ \begin{array} { l } 
{ x _ { 1 } } \\
{ y _ { 1 } } \\
{ z _ { 1 } }
\end{array} ] ) ^ { H } \left[k_{x}^{\prime}, k_{y}^{\prime}, k_{z}^{\prime}\left(\frac{N-1}{N}\left[\begin{array}{l}
x_{1} \\
y_{1} \\
z_{1}
\end{array}\right]-\frac{1}{N}\left[\begin{array}{l}
x_{2} \\
y_{2} \\
z_{2}
\end{array}\right] \ldots-\frac{1}{N}\left[\begin{array}{l}
x_{N} \\
y_{N} \\
z_{N}
\end{array}\right]\right)+\right.\right. \\
& \left(\left[k_{x}^{\prime}, k_{y}^{\prime}, k_{z}^{\prime}\right]\left[\begin{array}{c}
x_{2} \\
y_{2} \\
z_{2}
\end{array}\right]\right)^{H}\left[k_{x}^{\prime}, k_{y}^{\prime}, k_{z}^{\prime}\left(-\frac{1}{N}\left[\begin{array}{l}
x_{1} \\
y_{1} \\
z_{1}
\end{array}\right]+\frac{N-1}{N}\left[\begin{array}{c}
x_{2} \\
y_{2} \\
z_{2}
\end{array}\right] \ldots-\frac{1}{N}\left[\begin{array}{c}
x_{N} \\
y_{N} \\
z_{N}
\end{array}\right]\right)+\ldots\right. \\
& \left([ k _ { x } ^ { \prime } , k _ { y } ^ { \prime } , k _ { z } ^ { \prime } [ \begin{array} { c } 
{ x _ { N } } \\
{ y _ { N } } \\
{ z _ { N } }
\end{array} ] ) ^ { H } \left[k_{x}^{\prime}, k_{y}^{\prime}, k_{z}^{\prime}\left(-\frac{1}{N}\left[\begin{array}{c}
x_{1} \\
y_{1} \\
z_{1}
\end{array}\right]-\frac{1}{N}\left[\begin{array}{l}
x_{2} \\
y_{2} \\
z_{2}
\end{array}\right] \ldots+\frac{N-1}{N}\left[\begin{array}{c}
x_{N} \\
y_{N} \\
z_{N}
\end{array}\right]\right)=\right.\right. \\
& =\left(d x_{1}+d y_{1}\right)\left(\begin{array}{l}
\left(\frac{N-1}{N} d x_{1}-\frac{1}{N} d x_{2} \ldots-\frac{1}{N} d x_{N}\right)+ \\
\left(\frac{N-1}{N} d y_{1}-\frac{1}{N} d y_{2} \ldots-\frac{1}{N} d y_{N}\right)
\end{array}\right)+\ldots
\end{aligned}
$$

$$
\begin{gathered}
+\left(d x_{2}+d y_{2}\right)\left(\begin{array}{l}
\left(-\frac{1}{N} x_{1}+\frac{N-1}{N} d x_{2} \ldots-\frac{1}{N} d x_{N}\right)+ \\
\left(-\frac{1}{N} d y_{1}+\frac{N-1}{N} d y_{2}-\frac{1}{N} d y_{N}\right)
\end{array}\right)+\ldots \\
\left(d x_{N}+d y_{N}\right)\left(\begin{array}{l}
\left(-\frac{1}{N} d x_{1}-\frac{1}{N} d x_{2} \ldots+\frac{N-1}{N} d x_{N}\right)+ \\
\left(-\frac{1}{N} d y_{1}-\frac{1}{N} d y_{2} \ldots+\frac{N-1}{N} d y_{N}\right)
\end{array}\right)
\end{gathered}
$$

\section{REFERENCES}

[1] R.D. Chetan, A.N. Jadhav, "Simulation study on DOA estimation using MUSIC algorithm," Intl. J. Tech. Eng. Sys., vol. 2, no. 1, Mar. 2011.

[2] K. Ikeda, J. Nagai, T. Fujita, H. Yamada, A. Hirata, and T. Ohira, "DOA estimation by using MUSIC algorithm with a 9-elements rectangular ESPAR antenna," Proc. of Intl. Symp. on Antennas and Propagat., Aug. 2004, pp. 45-48.

[3] Chen Sun, and N.C. Karmakar, "Direction of arrival estimation based on a single port smart antenna using MUSIC algorithm with periodic signals,". Intl. J. Signal Process., vol. 1, no. 3, pp. 153-162.

[4] R.O. Schmidt, "Multiple emitter location and signal parameter estimation," IEEE Trans. Antennas Propagat., vol. 34, pp. 276-280, 1986.

[5] F.A. Belhoud, R.M. Shubair, and M.E. Al-Mualla, "Modelling and performance analysis of DOA estimation in adaptive signal processing arrays," Proc. IEEE Intl. Conf. on Electron., Circuits and Sys., Dec. 2003, pp. 340-343

[6] J.A. Cadzow, "A high resolution direction-of-arrival algorithm for narrow-band coherent and incoherent sources," IEEE Trans. Acoust., Speech, Signal Process., vol. 36, no. 7, pp. 965-979, Jul. 1998.

[7] A. Abouda, H.M. El-Sallabi, and S. G. Haggman, "Impact of antenna array geometry on MIMO channel eigenvalues," Proc. IEEE Intl. Symp. on Personal, Indoor and Mobile Radio Comm., vol. 1, Sep. 2005, pp. 568-572.

[8] Yu.B. Nechaev (2018, sep.). Estimation of the Cramer-Rao Bound for Radio Direction-Finding on the Azimuth and Elevation of Planar Antenna Arrays of the Symmetric Form . 2018 IEEE East-West Design \& Test Symposium (EWDTS). DOI: 10.1109/EWDTS.2018.8524799.

[9] B. Wu (2006). Realization and simulation of DOA estimation using MUSIC algorithm with uniform circular arrays. The 4th Asia-Pacific Conf. on Environmental Electromagnetics, pp. 908-912.

[10] Yu. B. Nechaev, I. V. Peshkov, "Evaluating Cramer-Rao Bound for 2D direction-finding via planar antenna arrays," Visn. NTUU KPI, Ser. Radioteh. radioaparatobuduv., no. 67, pp. 12-17, 2016.

[11] Gazzah, Houcem \& Marcos, Sylvie. (2006). Cramer-Rao bounds for antenna array design. Signal Processing, IEEE Transactions on. 54. 336 345. 10.1109/TSP.2005.861091.

[12] Baysal, Ülkü \& Moses, Randolph. (2003). On the geometry of isotropic arrays. Signal Processing, IEEE Transactions on. 51. 1469 - 1478. 10.1109/TSP.2003.811227.

[13] Thang Vu, Dinh \& Renaux, Alexandre \& Boyer, Rémy \& Marcos, Sylvie. (2011). A Cramér Rao bounds based analysis of 3D antenna array geometries made from ULA branches. Multidimensional Systems and Signal Processing. 24. 1-35. 10.1007/s11045-011-0160-5.

[14] Yang, Xiaopeng \& Long, Teng \& Sarkar, Tapan. (2010). Effect of geometry of planar antenna arrays on Cramer-Rao Bounds for DOA estimation. 389 - 392. 10.1109/ICOSP.2010.5657183.

[15] A.Y.J. Chan, J. Litva, "MUSIC and maximum likelihood techniques on two-dimensional DOA estimation with uniform circular array," IEE Proceedings - Radar, Sonar and Navigation, Vol.142, Iss. 3, pp. 105114.

[16] Yu.B. Nechaev, E. Algazinov, I. Peshkov (2018). Estimation of the Cramer-Rao Bound for Radio Direction-Finding on the Azimuth and Elevation of the Cylindical Antenna Arrays. 2018 41st International Conference on Telecommunications and Signal Processing (TSP), DOI: 10.1109/TSP.2018.8441419. 
[17] N. Dowlut and A. Manikas, "A polynomial rooting approach to superresolution array design," IEEE Trans. Signal Processing, vol. 48, pp. 1559-1569, June 2000.

[18] Gazzah, Houcem \& abed-meraim, Karim, "Optimum Ambiguity-Free Directional and Omnidirectional Planar Antenna Arrays for DOA Estimation," Signal Processing, IEEE Transactions, on. 57. 3942 - 3953. 10.1109/TSP.2009.2023943.

[19] V. M. Chiriac, Q. He, A. M. Haimovich and R. S. Blum, "Ziv-Zakai Bound for Joint Parameter Estimation in MIMO Radar Systems," in IEEE Transactions on Signal Processing, vol. 63, no. 18, pp. 49564968, Sept.15, 2015.

[20] Khan, D., \& Bell, K.L. Explicit Ziv-Zakai bound for DOA estimation with sparse linear arrays. 2009 3rd IEEE International Workshop on Computational Advances in Multi-Sensor Adaptive Processing (CAMSAP), 257-260.

[21] Lange, O., Bin Yang, (2011). Antenna geometry optimization for $2 D$ direction-of-arrival estimation for radar imaging. International ITG Workshop on Smart Antennas, Aachen, 2011, pp. 1-8. doi: 10.1109/WSA.2011.5741909.

[22] Ghani, A. Keyvani, F. Sedighy, S. H. (2018). Antenna array placement on limited bound for isotropic and optimal direction-of-arrival estimation, IET Signal Processing, 12, (3), p. 277-283, DOI: 10.1049/ietspr.2016.0472IET.

[23] Birinci, T., Tank, Y. (2007). Optimization of nonuniform array geometry for DOA estimation with the constraint on gross error probability. Signal Processing, Volume 87, Issue 10, Pages 2360-2369, https://doi.org/10.1016/j.sigpro.2007.03.012.

[24] Zhang, L. C. et al., (2014). L-Shaped Array Structure Optimization via Cramer-Rao Bound. Applied Mechanics and Materials, Vols. 556-562, pp. 3365-3368. https://doi.org/10.4028/www.scientific.net/AMM.556562.3365 .

[25] H. Moriya, etc., "Novel 3-D Array Configuration based on CRLB Fromulation for High-Resolution DOA Estimation," Proceedings of ISAP 2012, Nagoya, Japan, pp.1140-1143.

[26] Y. Hua, T.K. Sarkar, D.D. Weiner, "An L-shaped array for estimating 2D directions of wave arrival”, IEEE Trans. Antennas Propag.,V. 44. P. 889-895. Jun. 1996.

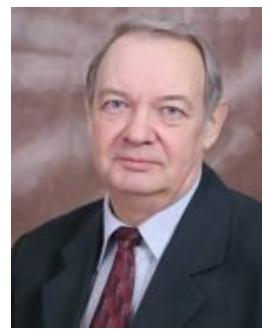

Yuri Nechaev was born in city of Khabarovsk which placed in the Far-East of the USSR, in 1944. $\mathrm{He}$ received the B.S. and M.S. degrees in radio physics from the Voronezh State University, USSR, Voronezh, in 1973 and awarded a doctorate in radio physics from Voronezh University, in 1995. From 2001 to 2004, he was with Concern Sozvezdie OJSC, Chief Researcher, Professor. He is now with Department of Information Systems, Voronezh State University. He is the author of more than 300 works, including 5 monographs, 8 textbooks and teaching aids, 18 copyright certificates and patents. His research interests include the development of the electrodynamics theory of microstrip antennas and microwave devices, the creation of new antenna structures and microwave devices for mobile radio communication systems, their introduction in industry. Dr. Nechaev was held by Diploma of the Main Department of Education of the Voronezh Region for the best scientific work (2001), Winner of the Voronezh Regional Prize in Science and Education (2002), Acknowledgments from the Head of the Administration of the Voronezh Region for their great contribution to the development of science and education (2004, 2005), Title "Honorary Machine Builder" (2005), Honorary Diploma for many years of creative management of the section "Electrodynamics, Radio Wave Propagation, Antenna" of the International Scientific and Technological Complex "Radiolocation, Navigation, Communication" (2006), Title "Honored Scientist" of Russia Federation (2008).

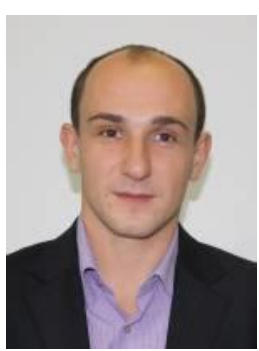

Ilia Peshkov was born in city of Yelets, Lipetskaya oblast, Russia in 1986. He received the B.S. degree in radio electronics and its service from Yelets State University named I.A. Bunin, Yelets, Russia, in 2008 and the $\mathrm{PhD}$ degree in radio physics from Voronezh State University, Voronezh, Russia, in 2012. From 2008 to 2013, he was a Research Assistant with the Voronezh State University, Voronezh, Russia and Concern Sozvezdie OJSC. Ilia Peshkov is now with Department of Physics, Radio engineering and Electronics, Bunin Yelets State University. His research interest includes: digital signal processing, electronics, digital diagramming and direaction-of-arrival estimation with super-resolution. Mr. Peshkov awards and honors include Honorary Certificate of the Department of Education and Science of the Lipetsk Region" for the development of Russian science, a great contribution to the organization of scientific research and many years of fruitful pedagogical activity". 